\title{
Trypanosoma cruzi-induced host immune system dysfunction: a rationale for parasite immunosuppressive factor(s) encoding gene targeting
}

\author{
Ali Ouaissi, ${ }^{1 \dagger}$ Anabela Cordeiro Da Silva, ${ }^{2}$ Angel Gustavo Guevara, ${ }^{3}$ \\ Margarida Borges, ${ }^{1}$ and Eliane Guilvard ${ }^{1}$ \\ ${ }^{1}$ CJF INSERM n $n^{\circ}$ 96-04, Centre IRD de Montpellier, 911 Av. Agropolis, BP 5945, 34032 Montpellier cédex 1, France \\ ${ }^{2}$ Department of Biochemistry, Faculty of Pharmacy, University of Porto, Portugal \\ ${ }^{3}$ Laboratory of Clinical Investigations, Vozandes Community Services, \\ Hospital Vozandes, Villalengua 267 y 10 de Agosto, Casilla 17-17-691, Quito, Ecuador
}

\begin{abstract}
An intense suppression of $\mathrm{T}$ cell proliferation to mitogens and to antigens is observed in a large number of parasitic infections. The impairment of T cell proliferation also occurred during the acute phase of Chagas' disease, caused by the intracellular protozoan parasite Trypanosoma cruzi. A wealth of evidence has accumulated that illustrates the ability of $T$. cruzi released molecules to influence directly a variety of diverse immunological functions. In this paper, we review the data concerning the immunoregulatory effects of T. cruzi Tc24 (a B cell activator antigen) and Tc52 (an immunosuppressive protein) released molecules on the host immune system. The gene targeting approach developed to further explore the biological function(s) of Tc52 molecule, revealed interesting unexpected functional properties. Indeed, in addition to its immunusuppressive activity a direct or indirect involvement of Tc52 gene product alone or in combination with other cellular components in T. cruzi differentiation control mechanisms have been evidenced. Moreover, targeted Tc52 replacement allowed the obtention of parasite mutants exhibiting low virulence in vitro and in vivo. Thus, the generation of a complete deficiency state of virulence factors by gene targeting should provide a means to assess the importance of these factors in the pathophysiological processes and disease progression. It is hoped that such approaches might allow rational design of tools to control T. cruzi infections.
\end{abstract}

\section{INTRODUCTION}

The ability of parasites to survive in the immune hosts depends upon a variety of escape mechanisms. One of these is the inhibition or the suppression of the immune response of their hosts. Several possible explanations have been put forward, such as antigenic competition, acquired tolerance, as well as the possible blocking role of soluble antigens or circulating immune complexes. The release by the parasites themselves of excretory-secretory products which have potent immunosuppressive activity represents another possible explanation. Moreover, a large number of reports have described the presence of suppressor cells ( $\mathrm{T}$ lymphocytes and macrophages) in humans and animals infected with various parasites.

In the case of Chagas' disease which remains a medical problem of relative important proportions in Latin America studies of the pathogenesis and immunology of the disease aiming to disclose the molecular mechanisms underlying the immunological alterations associated with T. cruzi infection are currently conducted in several laboratories over the world.

Trypanosoma cruzi, the etiological agent of Chagas' disease, is an obligate intracellular parasite causing chronic infections in human and a large number of other mammalian species [1]. This protozoan parasite is transmitted to man and other vertebrate hosts in the faeces of haematophagous bugs of the Reduviid family. The complex life cycle of T. cruzi includes different stages in the insect vector and the vertebrate host. There are two parasite stages in the vector: epimastigotes and metacyclic trypomastigotes, whereas the vertebrate stages are bloodstream trypomastigotes and intracellular amastigotes. Chagas' disease is associated with many immunological and immunopathological reactions. In fact, during either the chronic or the acute phase, various autoimmune phenomena were observed and could be the results of nonspecific polyclonal activation or suppressive effects that occur during T. cruzi infection. The existence of antigens cross-reactive between $T$. cruzi and mammalian cells was also reported as 
a possible cause of exacerbation of pathological manifestations [1].

\section{POLYCLONAL ACTIVATION}

The acute and chronic phases of a Trypanosoma cruzi infection are characterized by extensive polyclonal $\mathrm{B}$ and $\mathrm{T}$ cell activation [2]. In the early stages of infection, the number of immunoglobulin-secreting cells in the spleen and peripheral lymph nodes is very high and the majority of activated $B$ cell secrete antibodies nonspecific for the parasite antigens. These responses lead to the expansion of antiself clones that may be responsible for the killing of parasitized and nonparasitized cells. High cellularity (60\%-70\% of lymphocytes) is concomitant with a very large lymphocyte activation. In this phase of infection, the B cell polyclonal proliferation is characterized by a typical isotypic profile, IgG2a, and IgG2b, in the spleen and lymph nodes. Such responses are often described as polyclonal with a preferential production of these immunoglobulin isotypes, however these responses are not antigen specific. Spleen mRNA analysis of T. cruzi infected mice show an indiscriminate utilization of $\mathrm{VH}$ gene families with some D-proximal sets correlated with multireactivity. The hybridomas produced by spleen B cells or lymph nodes $\mathrm{T}$ cells of infected mice fail to bind parasite Ag but are autoreactive clones, in such a way that the antibodies recognize natural structures like actin, tubulin, myosin, keratin, myoglobulin, thyroglobulin, or myelin. T cells are also polyclonally activated and display high levels of cytolytic and helper cell activities, but the massive polyclonal activation is due to the minor lymphocyte subsets, namely CD5B and $\gamma \delta \mathrm{T}$ lymphocyte.

The depletion of $\mathrm{T}$ cell subpopulations in mice by treating with monoclonal anti-CD4 antibodies abolishes the increase of Ig secreting spleen cells. The elimination of T cells in the infected mice blocks the increase of $\operatorname{IgM}, \operatorname{IgG} 2 \mathrm{a}$, and IgG2b secreting spleen cells characteristic of the T. cruzi infection. Treatment of naive mice with the cell line G-05, isolated from lymph nodes of chronic infected mice, induces a $B$ cell polyclonal activation similar to that of chronic phase of infection [3]. Furthermore, recent studies have shown that immunization of mice with CL-14 trypomastigotes generated a protective response mediated by CD8 cells but does not induce polyclonal lymphocyte activation, immunosuppression, or pathology [3].

\section{Tc24 parasite released molecule behaves as a B-cell activator}

Like others microorganisms, T. cruzi displays mitogenic activities that induce the production of a large percentage of B lymphocytes in a nonspecific manner. The isolation of lipopolysaccharide (LPS)-like substances from T. cruzi has been reported [4]. Furthermore, we have shown that an octadecapeptide derived from the primary sequence of an $85 \mathrm{kDa}$ parasite surface protein could amplify the proliferative response of presensitized cells and could induce the production of antibodies against heterologous antigens in the peptide-immunized mice, thus acting as a comitogenic molecule [5]. Moreover, our recent investigations on the immunogenicity of a T. cruzi Tc24 released protein has revealed that in vivo treatment with the recombinant $\mathrm{Tc} 24$ protein induces a quick increase in the number of B cell secreted immunoglobulins mainly of $\operatorname{IgM}$ isotype. The $\operatorname{IgM}$ response is mostly unrelated to the antigens present in total parasite extracts or to the protein itself. Our results using euthymic and athymic mice are suggestive of a mitogenic activity of Tc24 on $\mathrm{B}$ cells, that is, $\mathrm{T}$ cell independent [5].

Tc24 induces a strong B-lymphocyte response using in vitro and in vivo assays, indicating that the nature of the B cell responses following Tc24 injection is indeed the polyclonal expansion of nonspecific, nonparasite directed B cell clones, similarly to other mitogen stimulations. A single injection of Tc24 without any adjutants induces the production of immunoglobulins that are not specific for parasite antigens, as described for other mitogenic products released by several microorganisms like bacteria, viruses, and parasites.

Thus, although acute infection with T. cruzi results in an intense polyclonal activation of both $\mathrm{T}$ and $\mathrm{B}$ cells, paradoxically, $\mathrm{T}$ cells isolated at the acute phase of infection show a marked state of unresponsiveness. A variety of mechanisms involved in $\mathrm{T}$ cell suppression have been described. Taking into account the large number of data reported until now, it is reasonable to assume that certain molecules contained in, or released by T. cruzi, could be primarily responsible for the host immune system dysfunction.

\section{IMMUNOSUPPRESSION}

In mice and humans, the acute phase of Chagas' disease is marked by a state of immunosuppression [1]. In vitro activated $\mathrm{T}$ cells from infected mice show a lower IL-2 production and proliferative response to mitogens, characteristic of the immunosuppressive response. The immunodeficiency characteristic of the acute phase of infection in mice is revealed by a lower humoral response against specific and nonspecific parasite antigens. In mice, the activity of parasite molecules and the immunosuppressive cells have been directly implicated in the immunodeficiency [1]. In vitro assays have demonstrated that the proliferative CD4 cell response against mitogens is high in the absence of CD8 or macrophages, but lower than the control. In CD8 knockout mice, the activation of CD4 cells is deficient, indicating that CD8 populations are involved in this immunosuppression. In fact, the notion of suppressive cell populations ( $\mathrm{T}$ cells, NK cells or macropahges) being involved in the immunosuppression phenomenon occurring during Chagas' disease, has been already documented by several investigators [1].

Moreover, the presence of parasite suppressive factors has been also reported. Indeed, it has been shown that a T. cruzi antigen molecule SAPA (Shed acute phase antigen) exhibiting a neuraminidase-transsialidase activity down-regulated $\mathrm{T}$ lymphocyte proliferation as a consequence of $\mathrm{T}$ suppressor/cytotoxic cell activation and secretion of PGE2, an immunoregulator effector substance [6]. Furthermore, recent studies have demonstrated that a T. cruzi membrane glyco- 
protein could inhibit the expression of IL-2 receptor chains and secretion of cytokines by subpopulations of activated human T lymphocytes [7].

\section{Tc52 parasite released molecule suppresses human and murine $T$-cell proliferation}

In recent reports we have cloned and sequenced a novel T. cruzi cDNA encoding a parasite released protein (named Tc52) containing a tandemely repeated structure characteristic of glutathione S-transferase [8]. Studies in serum containing culture system showed that native Tc52 had no mitogenic effect for human or murine $\mathrm{T}$ cells, instead Tc52 preparation at $1-4 \mu \mathrm{g} / \mathrm{ml}$ inhibited blastogenesis induced by antiCD3 antibodies or ConA [9]. This inhibition was not due to endotoxin, since the Tc52 preparations contained endotoxin at less than $0.1 \mu \mathrm{g} / \mathrm{ml}$, and endotoxin itself did not inhibit cell proliferation. The cells were not killed by Tc52 but were inhibited from responding throughout the culture period ( 3 to 4 days). The earlier the Tc52 was added the more inhibition was noted. In fact, Tc52 had little suppressive activity on lymphocyte proliferation once immune responses are initiated. Indeed, treatment of responding cells with Tc52 at least one hour before addition of triggering agents was needed to obtain an antiproliferative effect.

\section{MECHANISM(S) OF ACTION}

\section{TC52 as a scavenger of GSH and cysteine}

Tc52 native protein was eluted from S-hexyl glutathione affinity column by using glutathione (GSH) or cysteine and not other amino acids including those in GSH (e.g., glutamic acid and glycine) (Figure 1).We have hypothesized that $\mathrm{T}$ cell response could be affected by Tc52 through its interaction with GSH and cysteine. Indeed, it is interesting to remind that the diverse responses of T cells appear to involve a complex series of secondary signals in addition to the triggering of $\mathrm{T}$ cell antigen specific receptor. Moreover, it has been shown that the extracellular concentration of cysteine could be a limiting factor for T cell activation [11]. Indeed, the plasma cysteine concentration is 10 times lower than that of its oxidized form cystine. In addition, the $\mathrm{T}$ cell membrane transport for cystine is 10 times less efficient than for cysteine. In contrast, macrophages have the capacity to take up cystine efficiently and release cysteine, therefore increasing the delivery of this amino acid to $\mathrm{T}$ cells which in turn could increase their own GSH intracellular level [20].

The data we have reported provide clear evidence indicating that Tc52 which specifically interact with GSH and its precursor cysteine, almost completely inhibited human and mice lymphocyte proliferation in response to mitogens. This inhibition was completely reversed by addition of exogenous GSH or cysteine at physiological concentrations, but not methionine or other amino acids. Moreover, cells from T. cruzi-infected mice recovered during the time of active

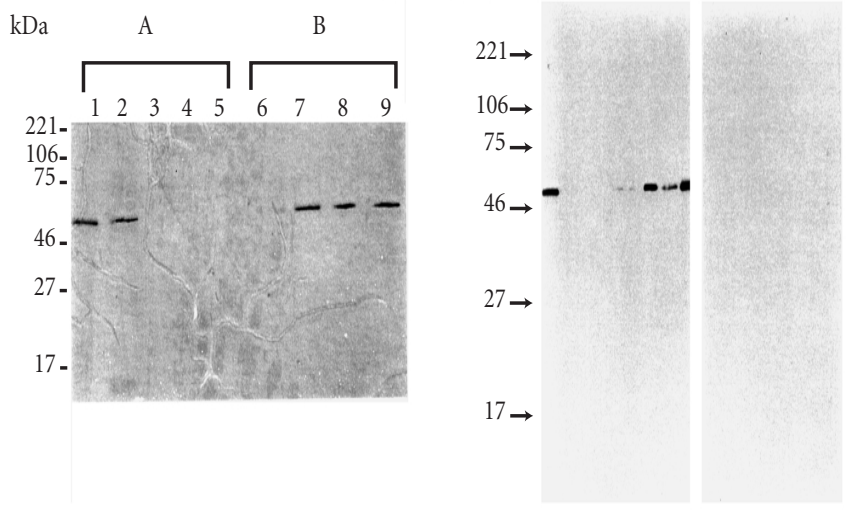

Figure 1: (A) Elution profiles of T. cruzi Tc52-bound to S-hexyl glutathione columns with either S-hexyl glutathione (1) or the following amino acids: L-cysteine (2), L-methionine (3), L-glycine (4), glutamic acid (5). (B) After elution as described in A, the columns from which the eluates analysed in lanes 2, 3, 4, and 5 were recovered, washed with the column buffer, and loaded with the same buffer containing S-hexyl glutathione, the corresponding eluates were analysed in lanes 6,7,8, and 9, respectively. As shown in lane 6, residual Tc52 could be recovered from the column which was first loaded with L-cysteine (lane 2). In contrast, Tc52 which still bound to the glutathione matrix after the elution with L-methionine (3), L-glycine (4) or Glutamic acid (5), could be efficiently eluted by S-hexyl glutathione (lanes 7, 8, 9, respectively). (C) Aliquots of the eluted samples in A (Ia) and B (IIa) (the lanes 1, 2, 3, 4, 5, 6, 7, and 8 , corresponded to the products eluted and analyzed in lanes 2,3 , $4,5,6,7$, and 9, respectively), were separated by SDS-PAGE, transferred to nitrocellulose filters and probed using a rabbit immune serum to Tc52 fusion protein (cDNA encoding the Tc52 protein, initially named TcAc2, was subcloned in pGEX-2T vector, and the protein was produced in fusion with Schistosoma japonicum $26 \mathrm{kDa}$ glutathione S-transferase (Tc52-Sj26GST)). The fusion protein and the corresponding antibodies were obtained as described in a previous report [10]. Ib and IIb represent control tests using samples analysed in Ia and IIa, respectively, and treated with anti-Sj26GST rabbit serum.

Methods: T. cruzi (Y strain) epimastigotes were maintained in culture medium as described in [10]. Parasite soluble antigens were prepared and adjusted to $5 \mathrm{mg}$ proteins/ $\mathrm{ml}$ in buffer $(20 \mathrm{mM}$ HEPES, $\mathrm{pH} 7.25,1 \mathrm{mM}$ EDTA, O.15 M KCl supplemented with $0.5 \mathrm{mM}$ final concentration of PMSF), and passed through S-hexyl glutathione affinity matrix. The column was then washed with $30 \mathrm{ml}$ of the same buffer, bound material was eluted using $20 \mathrm{ml}$ of buffer containing $2.5 \mathrm{mM}$ S-hexyl glutathione (1), or $1 \mathrm{mg} / \mathrm{ml}$ of either of the following amino acids: L-cysteine (2), L-methionine (3), L-glycine (4), or L-glutamic acid (5). The columns corresponding to the lanes $2,3,4$, and 5 were extensively washed with buffer and loaded with $20 \mathrm{ml}$ of buffer containing $2.5 \mathrm{mM}$ S-hexyl glutathione. All the eluates were dialysed against distilled water and analysed by SDS-PAGE. The proteins were visualized using the silver staining method. 
immunosuppression showed only moderate stimulation upon activation with anti-CD3 antibodies when compared to normal mouse spleen cells. Interestingly, the level of anti-CD3 stimulation increased significantly upon incubation of spleen cells from T. cruzi-infected mice with culture medium supplemented with cysteine or glutathione. Furthermore, Tc52 circulating in the blood of infected mice could be evidenced after 11 days of infection. Moreover, no antibodies against Tc52 could be detected during the acute phase of T. cruzi infection, whereas sera from chronically infected mice reacted against the parasite protein. It is well known that GSH deficiency could have dramatic consequences on the function of the immune system [11]. Moreover, T cells have been shown to be highly sensitive to low variations of extracellular concentrations of cysteine. Collectively, these data provide additional information that support the immunosuppression in Chagas' disease where Tc52, a T. cruzi released antigen which is a scavenger of GSH and cysteine, causes an alteration of T cell response.

\section{N-acetylcysteine (NAC) and glutathione modulate the behaviour of T. cruzi experimental infection}

According to the above observations, we have explored the efficacy of NAC or glutathione monoester (GSE), given orally to $T$. cruzi-infected mice, on T. cruzi clearance, T cell proliferation and $\mathrm{CD}^{+} / \mathrm{CD}^{+} \mathrm{T}$ cell ratios. The results obtained support the notion of a protective function of GSE or NAC against T. cruzi infection in BALB/c mice [6]. As expected, GSE, which is readily transported into cells and processed to GSH, seems to be more efficient than NAC. These data together with indications on the properties of NAC and GSE in different biological assays, suggest that the protective effects of GSE or NAC could be attributed to the immunomodulatory effects of these compounds. Up-regulation of the immune system by exogenous GSE or NAC may be useful to investigate further the benefit from therapeutic use of such components in combination with antiparasitic drugs against T. cruzi acute phase infection.

\section{TC52 modulates nitric oxide (NO) synthase and NO production by macrophages}

The preliminary data obtained suggested that Tc52 interferes with $\mathrm{T}$ cell activation through its capacity to scavenge glutathione and its precursor cysteine. However, recent studies have shown that GSH which constitutes the major source of plasma thiols and the most prevalent cellular thiol $(5-10 \mathrm{mM})$ is oxidized to GSSG upon its reaction with NO under both anaerobic and aerobic conditions [12]. In view of the fact that recent studies have shown that NO production by splenic macrophages interferes with $\mathrm{T}$ cell proliferation in mice infected with T. cruzi [13], we thought it would be interesting to investigate whether Tc52 can modify macrophage NO production and cytokine gene expression.

Our studies showed that Tc52 synergizes with IFN- $\gamma$ to stimulate NO production by macrophages [14]. The inducible $i N O S$ mRNA levels were also increased upon stimulation of macrophages with Tc52 and IFN- $\gamma$, suggesting a possi-

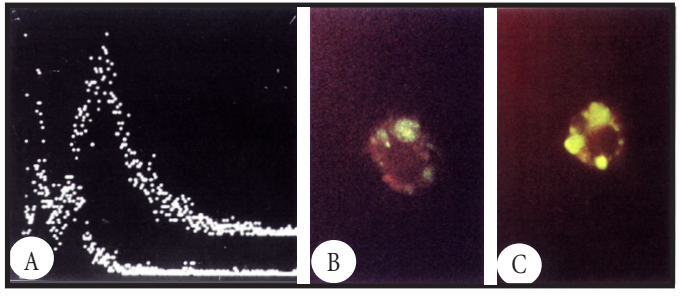

FIgure 2: Panel (A) shows the fluorescence intensity vs. relative cell number, obtained using $\mathrm{BALB} / \mathrm{c}$ mouse macrophages incubated at $4^{\circ} \mathrm{C}$ with Tc52 and treated with rabbit anti-Tc52 antibodies followed by fluoresceine-conjugated goat anti-rabbit Ig (right peak, 78\% fluorescent cells). The left peak represents cells which were incubated in the absence of Tc52 and treated with the antibodies as above (12\% fluorescent cells). Panel (B) shows that Tc52 "sticks" to macrophages upon its interaction with these cells at $37^{\circ} \mathrm{C}$. Panel (C) represents a high magnification of the same cell preparation after incubation with Tc52. The clumps of staining could be inside the cells or could be capped Tc52 that is on the upper surface of the cell.



FIGURE 3: Confocal analysis of mouse T. cruzi-infected macrophages (Mc) reacted with rabbit anti-Tc52 immune serum. Fluorescent intensity was converted into colors by the computer. Positive signals (large blue spots) are evidenced in association with T. cruzi intracellular amastigotes (A). (N), Macrophage nucleus.

ble control through transcriptional mechanisms. The mRNAs affected by Tc52 included IL- $1 \alpha$, IL-12, and IL-10. However, the combination of Tc52 and IFN- $\gamma$ down-regulates IL- $1 \alpha$ and IL-10 gene expression, but not IL-12. The Tc52 did not affect the expression of constitutively expressed gene encoding GAPDH. Complementary experiments showed that Tc52 could be bound to macrophage surface as evidenced by FACS analysis (Figure 2), and is expressed by intracellular amastigotes (Figure 3). The immunofluorescence micrographs of macrophages incubated with Tc52 protein may suggest a capping process. In fact the clumps of staining could be inside the cells or could be capped Tc52 that is on the upper surface of the cell (Figure 2). Further electron microscopy studies are required to determine how the molecule is taken by the cells (cell surface receptors?) and how it interacts to modify the intracellular molecular events leading to macrophage activation. 
The observations that exposure of macrophages to Tc52 induces the secretion of proinflammatory cytokines and NO indicate that in addition to a T-lymphocyte-mediated immune reactivity to Tc52, a direct effect of the exposure of mononuclear phagocytes to Tc52 must be considered in the pathogenesis of inflammation. In fact these inflammatory changes induced by Tc52 may contribute to protection against $T$. cruzi at an early stage of infection before an antigenspecific immune response is generated. Also, the synergistic activity of small amounts of IFN- $\gamma$ and Tc52 as observed in our studies indicates a protective role for Tc52 early during infection. However, the participation of NO in the suppression of $\mathrm{T}$ cell activation has been reported in a number of other biological systems [15]. Therefore, it is likely that NO production during the initial phase of acute T. cruzi infection might participate in the clearance of parasites by macrophages whereas its overproduction during the late phase of acute infection would account for the immunosuppression observed.

The involvement of parasite soluble factors in the downregulation of host $\mathrm{T}$ cell function is not restricted to T. cruzi but has been reported in a number of other parasite models such as Leishmania, Entamoeba histolytica, and Trypanosoma brucei (see ref. [14]). It is likely that these soluble factors can elicit a complex series of cellular interactions which result in specific immune responses or suppression depending on the immunoregulatory balance in the host. Thus, it is tempting to speculate that the parasite-derived factors(s) stimulate either directly or indirectly macrophages towards a suppressive phenotype.

One of the consequences of Chagas' disease is an inflammatory damage to myocardium and skeletal muscle [1]. Multiple mechanisms can lead to myocyte damage, including $\mathrm{CD}^{+}{ }^{+}$cytotoxic T cells, TNF- $\alpha$ cytotoxicity, autoantibodies to cardiac antigens, and NO production [16]. During the chronic phase of Chagas' disease, Tc52 produced by amastigotes inside T. cruzi infected cardiac endothelial cells and myocytes which have a transcriptionally $i N O S$, could modulate local production of NO and nitrotyrosine formation. These factors might participate in myocardial dysfunction and heart failure. To evaluate this possibility, we have recently developed transfection strategies of murine macrophages and fibroblasts with eucaryotic plasmid constructs containing the Tc52 encoding gene. This will enable investigations on the effects of intracellular production of Tc52 on NO synthesis and cytokine gene expression (Ouaissi et al., in preparation).

\section{DEVELOPMENT OF T. CRUZI ATTENUATED CLONES BY TC52 GENE REPLACEMENT}

Previous studies have shown that the degree of resistance to T. cruzi varied among mice strains (see ref. [14]). An additional factor which might potentially influence the course of disease and development or resistance is parasite strain virulence. Therefore, we have tested the feasibility of Tc52 gene replacement and analysed the phenotype of the genetically modified parasites in term of in vitro and in vivo parasite virulence. The results of our studies are encouraging. Indeed, monoallelic disruption of $T c 52\left(T c 52^{+} /^{-}\right)$resulted in the production of $T$. cruzi lines which express low capacity to proliferate in cultured mammalian cells and in BALB/c mice when compared to wild-type parasites. Surprisingly, there was a significant impairment of the ability of $\mathrm{Tc} 52^{+} \mathrm{I}^{-}$mutants or epimastigotes carrying a Tc52 antisense construct to go through their cycle of transformation into metacyclic trypomastigotes [17].

Several gene products that control the cell cycle in T. cruzi, such as the conserved cdc2-related kinases have been characterized. Previous studies have reported that the efficiency of epimastigote to metacyclic trypomastigote morphological transformation was significantly reduced in the case of noninfective clones and this correlates with a decrease of glucose6-phosphate dehydrogenase and 6-phosphogluconate dehydrogenase activities [18]. Moreover, it has been reported that competitive inhibitors of ADP-ribosyl transferase inhibited both the extracellular and the intracellular differentiation of T. cruzi amastigotes [19]. Since ADP-ribosyl transferase activity is widely required in the differentiation of higher animal cells and for efficient DNA repair, these findings may suggest that the enzyme regulates structural changes in DNA which may be involved in the repression and derepression of specific genes during parasite differentiation.

Our present finding raises an important question about the possible direct or indirect involvement of Tc52 gene product alone or in combination with other cellular components in T. cruzi differentiation control mechanisms. It is interesting to remind that Tc52 expresses a thiol transferase activity, and that the existence of a possible relationship between proteins involved in thiol-disulfide redox reactions and the regulation of gene expression is only partially emerging. For example, it is known that glutaredoxin are cofactors for ribonucleotide $[44,45]$. Furthermore, a functional glutaredoxin homologue identified in the vaccinia virus has been proposed to participate as a cofactor in the viral transcription to initiate gene expression through its capacity to mediate the thiol-disulfide conversion reactions [20]. Therefore, it would be not surprising if Tc52, like other members of the thiol-disulfide oxidoreductase family which act by shifting the balance between glutathione, disulfide glutahione, thiol groups of cysteines, and protein disulfide bonds plays a role in the regulation of gene expression. Further molecular in depth studies are needed to determine the involvement of Tc52 in T. cruzi gene expression regulation.

Another interesting observation related to the Tc52 down-modulation is the dramatic decrease in parasite virulence in vitro and in vivo, the reduction being even more pronounced in the case of parasites overexpressing antisense RNA, indicating therefore that we have generated mutant parasites with low virulence. The availability of the mutants obtained by gene targeting will allow to examine how the low virulence expressed by T. cruzi mutant influences the nature of the immune response during infection. Moreover, investigations on morphological changes occurring in tissues of mutant-challenged host will determine whether mutant parasites are incapable of causing disease. If that is the case then 
vaccination potential against subsequent challenge with virulent parasites have to be tested.

Similar strategy has already been applied in the case of Leishmania major and L. donovani strains for another gene, the Trypanothione reductase encoding gene, required for parasite survival [21]. Although, the TR mutant parasites have not been studied with respect to their ability to produce disease in experimental hosts, the data obtained clearly indicate decreased survival of the TR mutant inside macrophages in vitro. Thus, it is likely that the genetic manipulation approaches would not only help to define the in vivo role of a given gene product but may also be useful to develop stable attenuated strains for vaccine development.

\section{CONCLUDING REMARK}

Parasites can elicit a complex series of cellular interactions which result in specific immune responses or suppression depending on the immunoregulatory balance in the host. Suppression appears to predominate at certain phases of the infection. Relieving this parasite-induced immunosuppression could potentially provide a means to restore the efficacy of the immune protection mechanisms.

\section{ACKNOWLEDGMENT}

The studies reported in this paper received financial support from INSERM, Conseil Régional du Languedoc Roussillon and Fondation pour la Recherche Médicale. Margarida Coutinho is a recipient of a Portuguese Fundacao Para A Ciencia E A Tecnologia (Ministero Da Ciencia E Da Tecnologia) fellowship. A. G. G. is a recipient of a BID-FONDACYT (Ecuador) Chagas' disease Grant.

\section{REFERENCES}

[1] Takle GB, Snary D. South American trypanosomiasis (Chagas' disease). In: Kenneth S. Warren, ed. Immunology of Parasitic Infections. Blackwell Scientific Publications; 1993:213.

[2] Minoprio P, Itohara S, Heusser C, Tonegawa S, Coutinho A. Immunobiology of murine T. cruzi infection: the predominance of parasite-nonspecific responses and the activation of TcRI T cells. Immunol Rev. 1989;112:183.

[3] Paiva CN, Castelo-Branco MT, Lannes-Vieira J, Gattas CR. Trypanosoma cruzi: protective response of vaccinated mice is mediated by $\mathrm{CD} 8^{+}$cells, prevents signs of polyclonal $\mathrm{T}$ lymphocyte activation and allows restoration of a resting immune state after challenge. Exp Parasitol. 1999;91:7.

[4] Goldenberg S, Cordeiro MN, Silva-Pereira AA, MaresGuida L. Release of lipopolysaccharide (LPS) from cell surface of Trypanosoma cruzi by EDTA. Int J Parasitol. 1983;13:11.

[5] Cordeiro Da Silva A, Espinoza AG, Taibi A, Ouaissi A, Minoprio P. A 24000 MW Trypanosoma cruzi antigen is a B-cell activator. Immunol. 1998;94:189.

[6] Guevara AG, Guilvard E, Coutinho Borges M, Cordeiro Da Silva A, Ouaissi A. N-acetylcysteine and glutathione modulates the behaviour of T. cruzi experimental murine infections. Immunol Lett. 2000;71:79.

[7] Kierszenbaum F, de Diego JL, Fresno M, Sztein MB. Inhibitory effects of the Trypanosoma cruzi membrane glycoprotein AGC10 on the expression of IL2 receptor chains and secretion of cytokines by subpopulations of activated human T lymphocytes. Eur J Immunol. 1999;29:1684.

[8] Schöneck R, Marty B, Taibi A, et al. Trypanosma cruzi cDNA encodes a tandemly repeated domain structure characteristic of small stress proteins and glutathione S-transferases. Biol Cell. 1994;80:1.

[9] Ouaissi A, Guevara-Espinoza A, Chabe F, GomezCorvera R, Taibi A. A novel and basic mechanism of immunosuppression in Chagas' disease: Trypanosoma cruzi releases in vitro and in vivo a protein which induces $\mathrm{T}$ cell unresponsiveness through specific interaction with cysteine and glutathione. Immunol Lett. 1995;48:221.

[10] Pestel J, Deffort JP, Gras-Masse H, et al. Polyclonal cell activity of a repeat peptide derived from the sequence of an 85-kilodalton surface protein of Trypanosoma cruzi trypomastigotes. Inf Immun. 1992;60:715.

[11] Dröge W, Eck HP, Mihm S. HIV-induced cysteine deficiency and T-cell dysfunction - a rationale for treatment with N-acetylcysteine. Immunol Today. 1992;13:211.

[12] Hogg N, Singh RJ, Kalyanaraman B. The role of glutathione in the transport and catabolism of nitric oxide. FEBS Lett. 1996;382:223.

[13] Abrahamsohn IA, Coffman RL. Cytokine and nitric oxide regulation of the immunosuppression in Trypanosoma cruzi infection. J Immunol. 1995; 155:3955.

[14] Fernandez-Gomez R, Esteban S, Gomez-Corvera R, Zoulika K, Ouaissi A. Trypanosoma cruzi: Tc52 released protein-induced increased expression of nitric oxide synthase and nitric oxide production by macrophages. $J$ Immunol. 1998;160:3471.

[15] James SL. Role of nitric oxide in parasitic infections. Microbiol Rev. 1995;59:533.

[16] Rose NR. Myocarditis-from infection to autoimmunity. The Immunologist. 1996;4:67.

[17] Allaoui A, François C, Zemzoumi K, Guilvard E, Ouaissi A. Intracellular growth and metacyclogenesis defects in Trypanosoma cruzi carrying a targeted deletion of a Tc52 protein-encoding allele. Mol Microbiol. 999;32:1273.

[18] Alves AM, Tanuri A, De Almeida DF, Krüger WMA. Reversible changes in the isoenzyme electrophoretic mobility pattern and infectivity in clones of Trypanosoma cruzi. Exp Parasitol. 1993;77:246.

[19] Williams GT. Trypanosoma cruzi: inhibition of intracellular and extracellular differentiation by ADP-ribosyl transferase antagonists. Exp Parasitol. 1983;56:409. 
[20] Ahn BY, Moss B. Glutaredoxin homolog encoded by vaccinia virus in a virion-associated enzyme with thioltransferase and dehydrogenase reductase activities. Proc Natl Acad Sci USA. 1992;89:7060.

[21] Dumas C, Ouellette M, Tovar J, et al. Disruption of the trypanothione reductase gene of Leishmania decreases its ability to survive oxidative stress in macrophages. EMBO J. 1997;16:2590.

${ }^{\dagger}$ E-mail: ali . ouaissi@montp. inserm. fr 


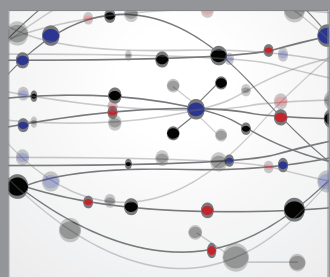

The Scientific World Journal
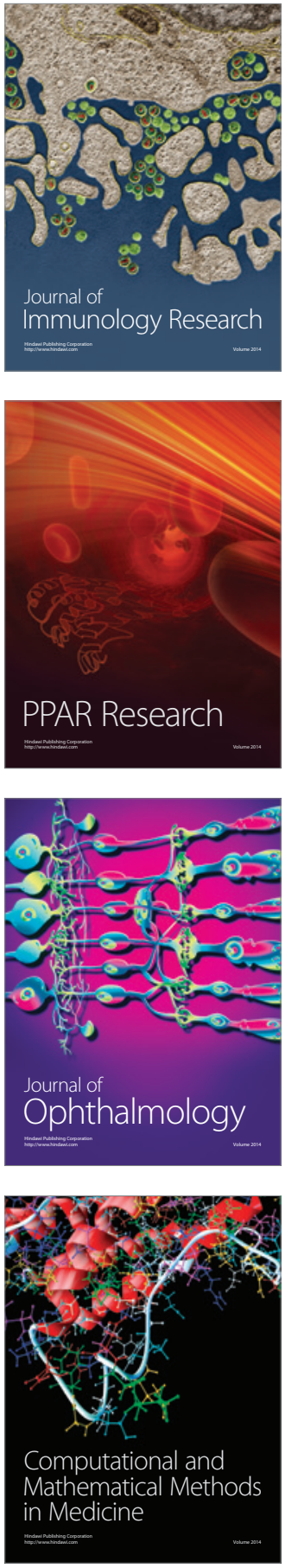

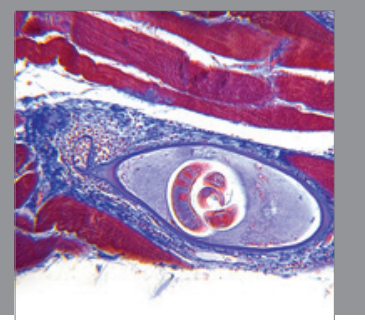

Gastroenterology

Research and Practice
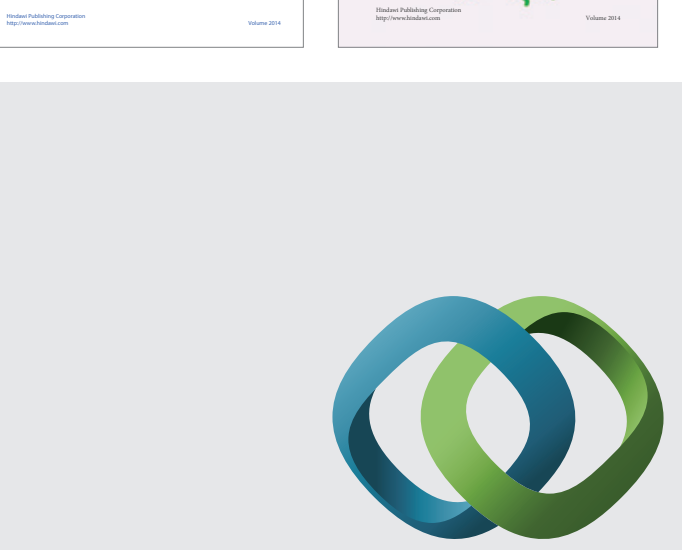

\section{Hindawi}

Submit your manuscripts at

http://www.hindawi.com
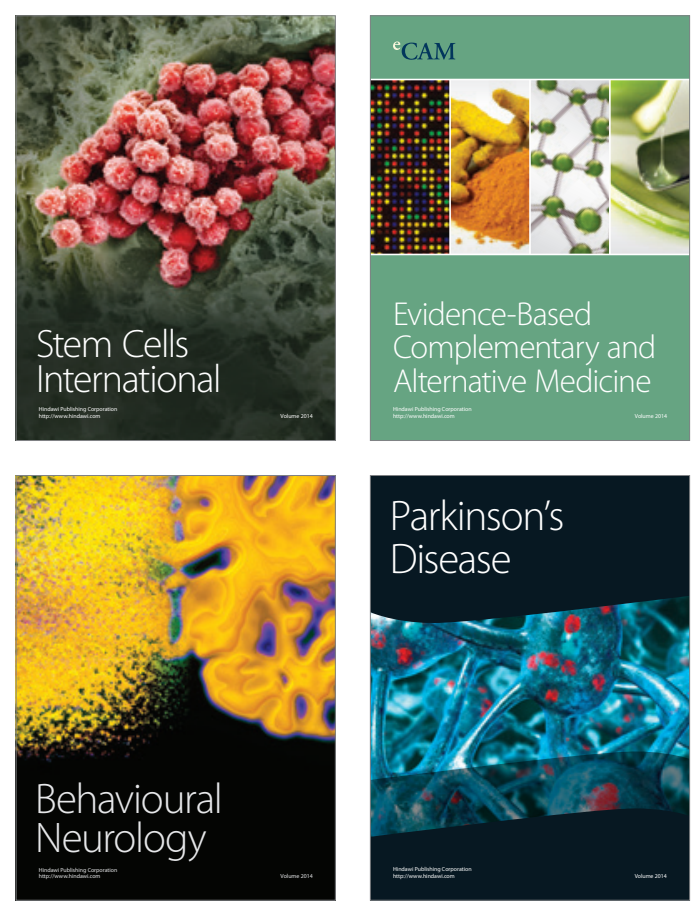

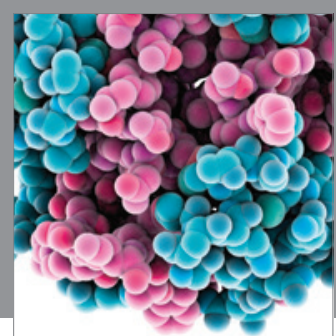

Journal of
Diabetes Research

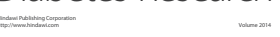

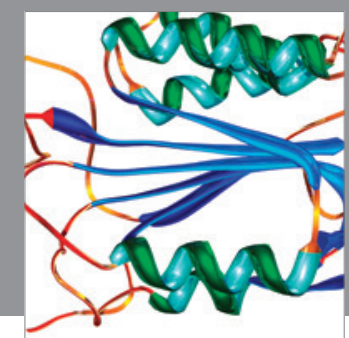

Disease Markers
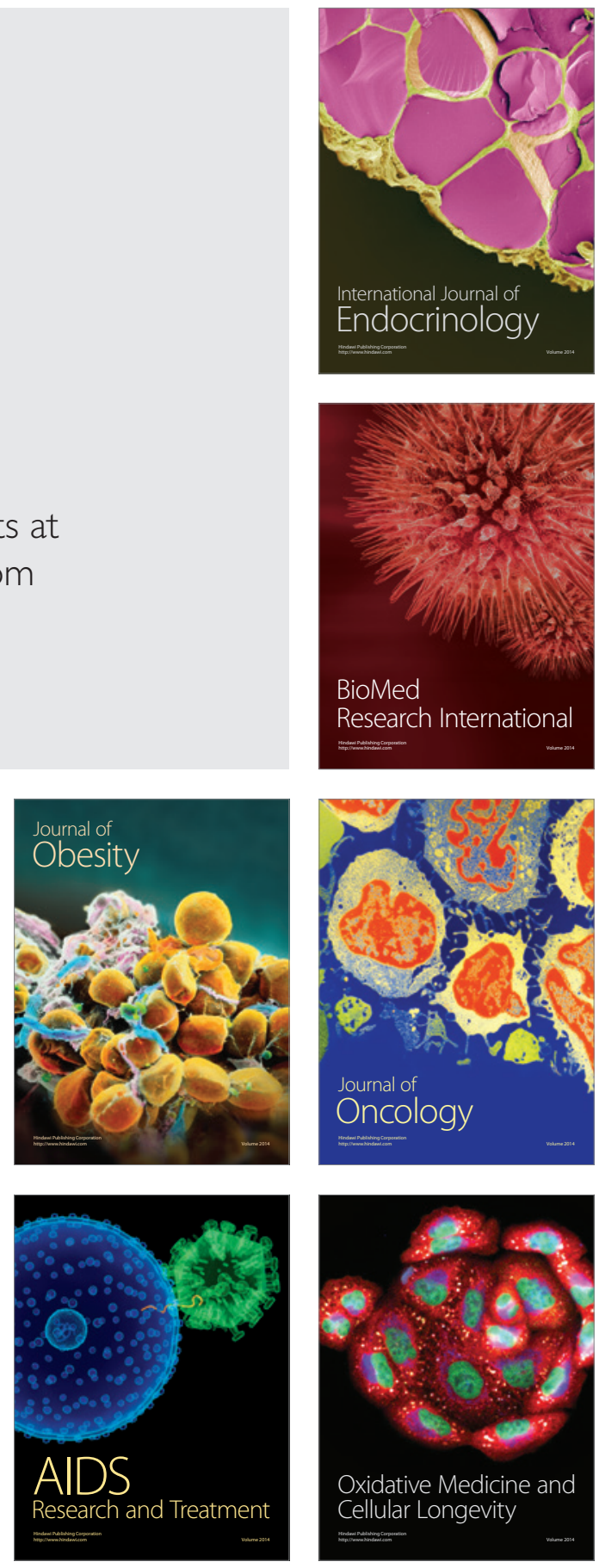